\title{
A GPIIb/IIIa bioreactor for specific treatment of immune thrombocytopenic purpura, an autoimmune disease. Preparation, in vitro characterization, and preliminary proof-of-concept animal studies
}

\author{
Emel Emregul, ${ }^{1 *}$ Allan David, ${ }^{1}$ Joseph P. Balthasar, ${ }^{2}$ Victor C. Yang ${ }^{1,3}$ \\ ${ }^{1}$ Department of Pharmaceutical Sciences, College of Pharmacy, University of Michigan, Ann Arbor, Michigan 48109-1065 \\ ${ }^{2}$ Department of Pharmaceutics, State University of New York at Buffalo, Buffalo, New York 14200
}

Received 1 April 2005; revised 13 May 2005; accepted 17 May 2005

Published online 17 August 2005 in Wiley InterScience (www.interscience.wiley.com). DOI: 10.1002/jbm.a.30470

\begin{abstract}
Immune thrombocytopenic purpura (ITP) is an autoimmune disease that affects thousands of Americans each year. The resulting thrombocytopenia, which develops from destruction of platelets (PLT) by anti-PLT autoantibodies (APAb), is often associated with hemorrhagic complications. Existing therapies are not effective and are associated with significant morbidity. Recently, a new treatment modality using plasmapheresis with a Protein-A column has shown some clinical promise. Yet, although this method would remove the pathogenic APAb, it would also deplete protective antibodies, thereby weakening the body's selfdefense system. Because about $80 \%$ of patients with ITP develop APAb against the GPIIb/IIIa antigens on PLT, a novel approach of attaching a GPIIb/IIIa-linked bioreactor with an extracorporeal circuit is suggested herein to achieve highly effective/specific APAb removal and overcome shortcomings of plasmapheresis in treating ITP. A hollow fiber-based bioreactor device was fabricated, and GPIIb/IIIa antigens were immobilized onto the inner lumens of the
\end{abstract}

hollow fibers by using the epichlorohydrin activation method. An optimized bioreactor containing a loading of $1.63 \mathrm{mg} \mathrm{GPIIb} / \mathrm{III} / \mathrm{g}$ fibers and adsorption capacity of 1.9 mg 7E3/g fibers was developed. Preliminary proof-of-concept investigation using a 7E3-induced thrombocytopenic rat model (which mimicked clinical ITP) was carried out. A complete $(100 \%)$ return of PLT counts to their initial levels was observed in rats within $6 \mathrm{~h}$ after the GPIIb/IIIa bioreactor treatment. In addition, a rapid restoration of WBC counts in the treated rats was also found. These preliminary findings shed light of promise of using the GPIIb/IIIa bioreactor approach in achieving highly improved ITP therapy. (C) 2005 Wiley Periodicals, Inc. J Biomed Mater Res 75A: 648-655, 2005

Key words: autoimmune disease; immune thrombocytopenic purpura (ITP); GPIIb/IIIa; hollow fiber-based bioreactor; plasmapheresis

\section{INTRODUCTION}

Immune thrombocytopenic purpura (ITP) is a common autoimmune disease with an estimated incidence rate of $10-125$ per million persons each year. ${ }^{1}$ Thrombocytopenia in ITP develops as a result of an enhanced destruction of platelets (PLT), which is precipitated by the interaction of autoantibodies and PLT antigens. ${ }^{2}$ The resulting thrombocytopenia is often dramatic and associated with a variety of hemorrhagic sequelae, including

Victor C. Yang is a Cheung Kong Scholar, School of Chemical Engineering, Tianjin University, Tianjin 300072, China.

*Present address: Faculty of Science, Department of Chemistry, University of Ankara, Ankara, Turkey 06110

Correspondence to: Victor C. Yang; e-mail: vcyang@ umich.edu

Contract grant sponsor: National Institutes of Health; contract grant numbers: R41 HL 59705 and R01 HL 67347 epistaxis, oral mucosal bleeding, petechiae, hematuria, gastrointestinal bleeding, and intracranial hemorrhage.

Currently accepted treatments for ITP include pharmacological immunosuppression, splenectomy, and intravenous immunoglobulin (IVIG) therapy, which includes administration of a heterogeneous pool of human IgG, IgM, and IgA. Unfortunately, pharmacological immunosuppression places patients at risk for the development of opportunistic infections and, for most patients, is not able to maintain platelet counts $>30 \times 10^{9} / \mathrm{L}$, the value typically accepted as sufficient to provide prophylaxis against severe hemorrhage. ${ }^{1}$ On the other hand, splenectomy is associated with considerable morbidity and incomplete efficacy, particularly in patients with chronic ITP. ${ }^{3}$ Subsequently, Imbach and coworkers reported that large doses of IVIG increases platelet counts in patients with ITP. ${ }^{4}$ The efficacy of IVIG was further confirmed by a number of additional studies. ${ }^{5-9}$ However, IVIG effects are 
typically short-lived, and the exceptionally high cost of this therapy prohibits the possibility for long-term applications to ITP patients with chronic disease. ${ }^{10,11}$ In addition, this IVIG treatment is beset by certain serious complications. ${ }^{12-18}$ Several new therapeutic modalities are currently under investigation for the treatment of ITP, including 1) application of Protein-A immunoadsorption by using a PROSORBA column ${ }^{19}$; 2) administration of recombinant thrombopoietin ${ }^{20,21}$; and 3) administration of immunoglobulin with a high titer of Anti-Rho (D) antibodies. ${ }^{22,23}$ Nevertheless, these experimental therapies have all encountered significant shortcomings and risks. For instance, given the evidence that antiplatelet antibodies (APAb) are responsible for the increased rate of PLT destruction observed in ITP, immunoadsorptive plasmapheresis has been developed to accelerate the removal of pathogen antibodies. Indeed, this method using the PROSORBA column has been approved by the Food and Drug Administration (FDA) for clinical use in treatment of ITP. ${ }^{19,24,25}$ The PROSORBA column is made of silica gel containing immobilized Protein-A, a bacterial protein that binds the Fc region of immunogammaglobulin with high affinity. ${ }^{26}$ Although this PROSRBA column removes pathological antibodies and immune complexes from the patient, it also depletes protective antibodies, thereby weakening the body's self-defense system and increasing patient susceptibility to opportunistic infections. In addition, Protein-A therapy has also been associated with a variety of thrombotic disorders including stroke. ${ }^{27,28}$

In this work, we proposed a novel bioreactor approach, designed to specifically remove APAb from blood, to overcome the limitations of extracorporeal $\mathrm{APAb}$ removal by the nonspecific Protein-A column. Because $>80 \%$ of patients with ITP develop antibodies directed against GPIIb/IIIa of PLT, ${ }^{29}$ GPIIb/IIIa was selected as the specific antigen for the development of this bioreactor.

The bioreactor developed herein consists of a hollow fiber-based device consisting of the GPIIb/IIIa antigens that are covalently coupled to the inner lumens of the hollow fibers by using the well-established epichlorohydrin activation method. ${ }^{30,31}$ Several design parameters were characterized to optimize the antigen-loading and adsorption capacity of the bioreactor. Preliminary in vivo studies involving rats were also carried out to provide proof-of-concept of this novel bioreactor-based approach to treat ITP.

\section{MATERIALS}

Cellulose hollow fibers were obtained by disassembling a Baxter Model CF 25 hemodialyzer (Baxter Healthcare Corporation, Deerfield, IL, USA). Each hollow fiber, with an internal diameter of $20 \mu \mathrm{m}$ and a wall thickness of $10 \mu \mathrm{m}$, is

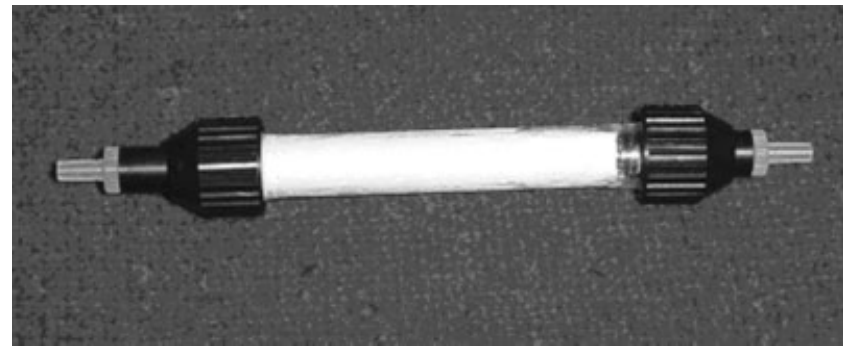

Figure 1. Schematic illustration of the fabricated bioreactor device.

made of a Cuprophan membrane with a 10-kDa molecular weight cutoff. Epichlorohydrin, ethanol amine, sodium hydroxide, and bromothymol blue were purchased from Sigma Chemical Co. (St. Louis, MO, USA). Human GPIIb/ IIIa was purchased from Enzyme Research Laboratories (South Bend, IN, USA). The monoclonal antiplatelet antibody, 7E3, was generously supplied by Dr. Joseph Balthasar at The State University of New York at Buffalo, New York. Buffers were prepared by using certified ACS reagents and in distilled, deionized water $\left(\mathrm{ddH}_{2} \mathrm{O}\right)$.

\section{METHODS}

\section{Fabrication of the bioreactor device}

The hollow fibers were cut to a length of $10 \mathrm{~cm}$, measured to a dry weight of $1 \mathrm{~g}$, and then bonded together with silicone. After allowing the silicone to dry and harden at room temperature, the tips of the fiber bundle were cut to remove any silicone blockage of the fiber openings. The bundle was then housed in a glass tube (inner diameter ca. $1.2 \mathrm{~cm}$ ) that is fitted with a luer connector at each end. Figure 1 presents an illustration of the bioreactor device. The prepared bioreactor possesses hemodynamic characteristics that mimic clinically used hemodialyzers.

\section{Activation of the hollow fiber-based bioreactor}

The hollow fiber-based bioreactor fabricated above was activated by circulating $10 \mathrm{~mL}$ of $0.8 \mathrm{M}$ sodium hydroxide, containing $0.5 \mathrm{~mL}$ epichlorohydrin, for $2 \mathrm{~h}$ at $40^{\circ} \mathrm{C}$. The bioreactor was then washed repeatedly with a large volume of $\mathrm{ddH}_{2} \mathrm{O}$ until no epichlorohydrin could be detected. To determine the presence of epichlorohydrin, $2 \mathrm{~mL}$ of flow through water was put into a glass tube followed by the addition of $1 \mathrm{~mL}$ of $1.3 \mathrm{M}$ sodium thiosulfate solution and 1 drop of bromothymol blue solution (indicator). A blue color indicated the presence of epichlorohydrin, whereas a yellow color signified a complete removal of this activating agent.

\section{Determination of epoxy groups on the activated hollow fibers}

Content of the epoxy groups on the epichlorohydrin-activated hollow fibers was determined by titration. ${ }^{32}$ Briefly, 
the hollow fiber bioreactor was first washed with $\mathrm{ddH}_{2} \mathrm{O}$. After removing the water, $1.3 \mathrm{M}$ sodium thiosulfate solution was circulated through the bioreactor for a half hour at room temperature. The epoxy content in the bioreactor was then determined by titration of the rinsed thiosulfate solution with $0.01 \mathrm{M}$ hydrochloric acid by using bromothymol blue as the indicator. The change of color from blue to yellow indicates the titration end point where the moles of hydrochloric acid consumed were equal to the moles of epoxy in the bioreactor.

\section{Coupling of GPIIb/IIIa onto the activated hollow fiber bioreactor}

GPIIb/IIIa solution $(0.5 \mathrm{mg} / \mathrm{mL}$ in $0.15 \mathrm{M}$ PBS buffer, $\mathrm{pH}$ 8.3) was circulated through the bioreactor for $12 \mathrm{~h}$ at $40^{\circ} \mathrm{C}$. The bioreactor was then washed with $0.15 M$ PBS buffer $(\mathrm{pH}$ 7.4). Unreacted epoxy groups were blocked by reacting with $1 \mathrm{M}$ ethanol amine, in $0.1 \mathrm{M}$ borate buffer at $\mathrm{pH} 8.0$, for overnight at room temperature. The bioreactor was then washed with $\mathrm{ddH}_{2} \mathrm{O}$, PBS buffer ( $\left.\mathrm{pH} 7.4\right), \mathrm{NaCl}(2 \mathrm{M})$, and then stored at $40^{\circ} \mathrm{C}$. The amount of coupled GPIlb/IIIa was determined by measuring the GPIIb/IIIa concentration in solution before and after circulation, according to the method of Bradford. ${ }^{33}$

\section{Coupling of albumin onto activated hollow fiber}

An albumin bioreactor was developed to assess the nonspecific binding of monoclonal antiplatelet antibody, 7E3. Albumin was coupled onto epichlorohydrin-activated hollow fibers according to the same procedures described above. The amount of immobilized albumin was determined by measuring the initial and final albumin concentrations in the coupling solution. ${ }^{33}$

\section{Leakage of coupled GPIIb/IIIa}

To determine the degree of leakage of GPIIb/IIIa from the bioreactor, PBS buffer (10 mL, pH 7.4) was circulated through the GPIIb/IIIa-coupled bioreactor at room temperature over a period of 4 days. The PBS elution was carried out, by using $100 \mu \mathrm{L}$ fresh PBS buffer, every 30 min for $3 \mathrm{~h}$ and then everyday for 4 days. Leakage was determined by measuring the GPIIb/IIIa concentration in the eluting solution by using the Bradford protein assay method. ${ }^{33}$ The degree of leakage was expressed as the percentage of eluted GPIIb/IIIa relative to the amount of immobilized GPIIb/ IIIa.

\section{In vitro adsorption test of monoclonal antiplatelet antibody (7E3)}

The adsorption of 7E3 antibodies on the GPIIb/IIIa bioreactor was examined in vitro. In brief, the bioreactor was washed with PBS buffer ( $\mathrm{pH}$ 7.4) at a flow rate of $5 \mathrm{~mL} / \mathrm{min}$.

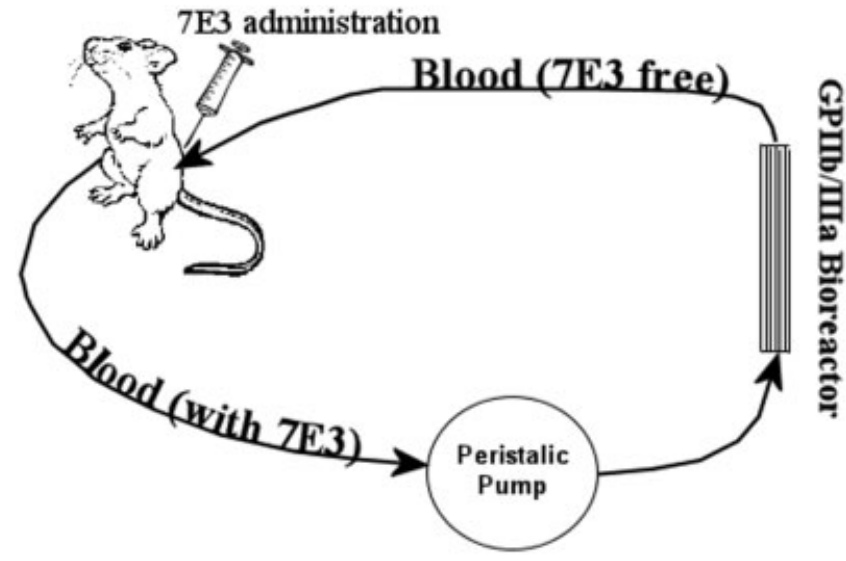

Figure 2. In vivo experimental setup.

The 7E3 antibody solution $(0.25 \mathrm{mg} / \mathrm{mL})$ was then pumped through the bioreactor at the same flow rate for $20 \mathrm{~min}$ at room temperature. The amount of 7E3 antibodies adsorbed onto the bioreactor was determined by measuring 7E3 concentrations before and after the adsorption process according to the method of Bradford. ${ }^{33}$ The adsorption of 7E3 onto a sham bioreactor was used as the control to assess the degree of nonspecific adsorption of 7E3 onto plain hollow fibers.

\section{Desorption of 7E3 and repeated uses of the bioreactor}

Desorption of 7E3 was studied by circulating a $0.1 M$ amino acetic acid solution through the 7E3-adsorbed GPIIb / IIIa bioreactor at room temperature for $2 \mathrm{~h}$. The final 7E3 concentrations in the eluant were determined.

To test the reusability of the GPIIb/IIIa bioreactor, the 7E3 adsorption-desorption procedure was repeated 8 times over a period of 40 days with the same bioreactor. Furthermore, repeated use of the GPIIb/IIIa bioreactor on the same day was investigated. Leakage of GPIIb/IIIa from the bioreactor after 7E3 desorption was also examined.

\section{Preliminary in vivo, proof-of-concept of the bioreactor approach in rats}

Female Sprague-Dawley rats, 200-250 g, were used for in vivo testing of the feasibility of the bioreactor approach. The aortal artery and the jugular vein of the rat were cannulated, and to the cannulas a hollow fiber bioreactor was attached to form a simple extracorporeal bypass loop. A dose of 4 $\mathrm{mg} / \mathrm{kg}$ of 7E3 was administered via the jugular vein cannula of the rat to induce thrombocytopenia. Control animals were dosed with an equal volume of sterile saline. Extracorporeal circulation through the bioreactor was initiated $1 \mathrm{~h}$ after administration of 7E3 (or saline), when platelet counts are expected to be $15-25 \%$ of initial values (i.e., in 7E3-treated animals). Heparin (250 mg/mL) was administered via the jugular vein cannula to prevent coagulation in the bioreactor and extracorporeal loop. Figure 2 provides a schematic of the in vivo experimental setup. 
Animals were tested in three groups: 1) a control group consisting of rats receiving 7E3; 2) a second control group of rats connected with an albumin-bioreactor and receiving 7E3; and 3) a treatment group of rats connected with the GPIIb/IIIa bioreactor and receiving the 7E3 antiplatelet antibody. Because this was a preliminary proof-of-concept investigation in animals, each group consisted of only three rats.

The blood was circulated through the hollow fiber device at a flow rate of $3 \mathrm{~mL} / \mathrm{min}$ for $1 \mathrm{~h}$. Platelet counts were determined in PLT rich plasma by using a Z1-Dual Coulter Counter fitted with a 70-mm aperture tube, just before dosing and at $1,3,6,12$, and $24 \mathrm{~h}$ after dosing. Concentrations of 7E3 were measured by using the ELISA assay. ${ }^{34}$

Animal studies were conducted at Dr. Balthasar's laboratory at the State University of New York at Buffalo (SUNYBuffalo). SUNY-Buffalo is fully accredited by the AAALAC, and the animal care and use program conforms to the standards in the "Guide for the Care and Use of Laboratory Animals," DHEW Pub. No. (NIH) 80-23, revised 1985.

\section{RESULTS AND DISCUSSION}

Activation of hydroxyl-containing polymers with epichlorohydrin in alkaline medium has been used widely for the preparation of adsorbents for bio-specific affinity chromatography. ${ }^{35}$ To optimize the functionality of the GPIIb/IIIa bioreactor, the effects of several parameters on the loading and adsorption capacity of the bioreactor were examined.

\section{Optimization of GPIIbIIIa immobilization}

Effects of epichlorohydrin concentration on hollow fiber activation

Solutions $(10 \mathrm{~mL})$ containing various concentrations of epichlorohydrin were circulated through the hollow fiber bioreactors at $40^{\circ} \mathrm{C}$ for $2 \mathrm{~h}$. Figure 3 shows the amount of epoxy groups on the bioreactor increased with increasing epichlorohydrin concentrations until a plateau was reached. Indeed, by using $1.0-\mathrm{g}$ hollow fibers, as much as $30 \mu \mathrm{M}$ hydroxyl groups could be activated when a $1.25 \%$ epichlorohydrin solution was used. The solution that contained $0.5 \%$ epichlorohydrin solution was found to yield sufficient activation (i.e., $26 \mu M$ epoxy per gram fiber) and, therefore, was used in subsequent experiments.

Effect of temperature and duration of coupling on GPIIb/IIIa loading

The GPIIb/IIIa coupling reaction was carried out under varying conditions as listed in Table I. The amount of GPIIb/IIIa coupled to the bioreactor de-

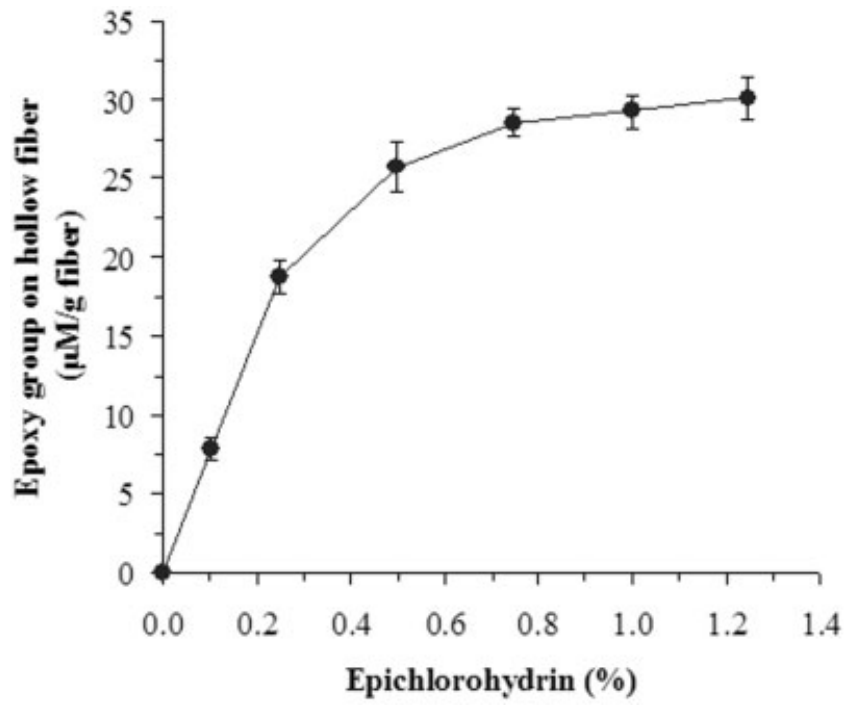

Figure 3. Effect of ephichlorohydrin concentration on the introduction of epoxy groups to the hollow fiber surface. Experimental details were provided in the Methods section. All measurements were performed in triplicate. Error bars represented standard deviation.

pended on both the coupling temperature and duration. Higher amounts of GPIIb/IIIa loading were observed as the coupling temperature and/or the coupling time are increased. A maximum of $1.624 \mathrm{mg}$ of GPIIb/IIIa per gram of fiber was obtained when the coupling reaction was carried out at $90^{\circ} \mathrm{C}$ over $6 \mathrm{~h}$. When conducted at $70^{\circ} \mathrm{C}$ over $6 \mathrm{~h}$, the coupling yield was approximately $85 \%$ of the maximum obtained at $90^{\circ} \mathrm{C}$. Although immobilized GPIIb/IIIa was found to be relatively stable under extreme experimental conditions, these exceedingly high temperatures (e.g., $>70^{\circ} \mathrm{C}$ ), nevertheless, introduced a significant degree of denaturation of the coupled GPIIb/IIIa (data not shown). On the other hand, room temperature was found to yield a low GPIIb/IIIa loading; $<23 \%$ of the maximum loading was attained even with coupling times as long as $72 \mathrm{~h}$. Therefore, the optimal conditions for GPIlb/IIIa coupling were selected at $40^{\circ} \mathrm{C}$ for $12 \mathrm{~h}$, simply because such conditions provided a yield of $90 \%$ compared to the maximum value. In addition, these conditions did not elicit any obvious impairment with regard to the binding affinity of the coupled GPIIb/IIIa toward 7E3.

\section{Effect of coupling $\mathrm{pH}$ on GPIIb/IIIa loading}

The optimum $\mathrm{pH}$ for GPIIb/IIIa coupling was examined by varying $\mathrm{pH}$ of the coupling buffers while the coupling conditions were set at $40^{\circ} \mathrm{C}$ for $12 \mathrm{~h}$. Figure 4 shows that there was a strong dependence between the amount of GPIIb / IIIa coupled and the $\mathrm{pH}$ in the coupling buffer. On the basis of these results, 
TABLE I

Effects of Temperature and Duration of Coupling on GPIIb/IIIa Loading

\begin{tabular}{cccr}
\hline $\begin{array}{c}\text { Temperature } \\
\left({ }^{\circ} \mathrm{C}\right)\end{array}$ & $\begin{array}{c}\text { Reaction } \\
\text { Time }(\mathrm{h})\end{array}$ & $\begin{array}{c}\text { Coupled } \\
\text { GPIIb/IIIa } \\
(\mathrm{mg} / \mathrm{g} \text { fibers })\end{array}$ & $\begin{array}{c}\text { Relative } \\
\%\end{array}$ \\
\hline 25 & 12 & 0.125 & 7.7 \\
& 72 & 0.366 & 22.5 \\
40 & 3 & 0.825 & 50.8 \\
& 6 & 1.150 & 70.8 \\
70 & 12 & 1.465 & 90.2 \\
90 & 6 & 1.380 & 85.0 \\
& 6 & 1.624 & 100.0 \\
\hline
\end{tabular}

the optimum $\mathrm{pH}$ for GPIIb/IIIa coupling was determined to be 8.3 .

Effect of concentration of coupling buffer on GPIIb/ IIIla loading

To evaluate the effect of the buffer concentration on coupling efficiency, PBS buffers ( $\mathrm{pH}$ 8.3) of varying concentrations were used while maintaining the coupling reactions at $40^{\circ} \mathrm{C}$ for $12 \mathrm{~h}$. Figure 5 shows that the optimum buffer concentration was $0.15 M$ PBS buffer.

Effect of GPIIb/IIIa concentrations on loading

The effects of the GPIIb/IIIa concentrations in the coupling solution on GPIIb/IIIa loading were studied in the range of $0-1 \mathrm{mg} / \mathrm{mL}$. Figure 6 shows that the amount of coupled GPIIb/IIIa increased with increasing GPIIb/IIIa concentration but reached a maximum at $0.5 \mathrm{mg} / \mathrm{mL}$. However, further increase in the GPIIb/IIIa concentration resulted in a decrease in the

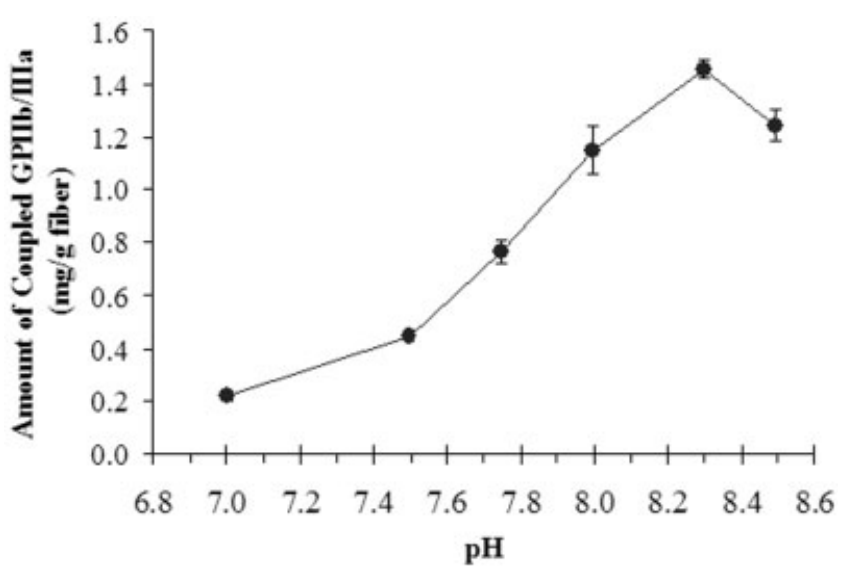

Figure 4. Effect of $\mathrm{pH}$ on coupling of GPIIb/IIIa to the bioreactor. Experimental details were provided in the Methods section. All measurements were performed in triplicate. Error bars represented standard deviation.

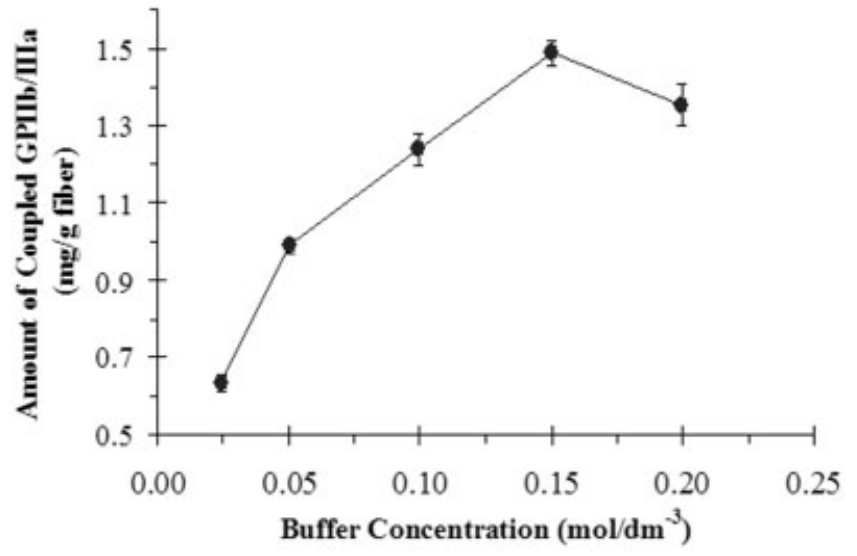

Figure 5. Effect of buffer concentration on coupling of GPIIb/IIIa. Experımental details were provided in the Methods section. All measurements were performed in triplicate. Error bars represented standard deviation.

amount of coupled GPIIb/IIIa, a phenomenon that was probably attributed to the high viscosity in the coupling solution. Therefore, the optimum GPIIB/IIIa concentration to be used in the coupling reaction was determined to be $0.5 \mathrm{mg} / \mathrm{mL}$.

\section{In vitro characterization of the GPIIb/IIIa bioreactor}

Adsorption and desorption of 7E3 antibodies by the GPIIb/IIIa bioreactor

To test the ability of the GPIIb/IIIa bioreactor in removing 7E3 antibodies, solutions containing 0.25 $\mathrm{mg} / \mathrm{mL} 7 \mathrm{E} 3$ were circulated through the bioreactors prepared in Figure 6 with different GPIIb/IIIa loadings at $37^{\circ} \mathrm{C}$ for $20 \mathrm{~min}$. The results also plotted in

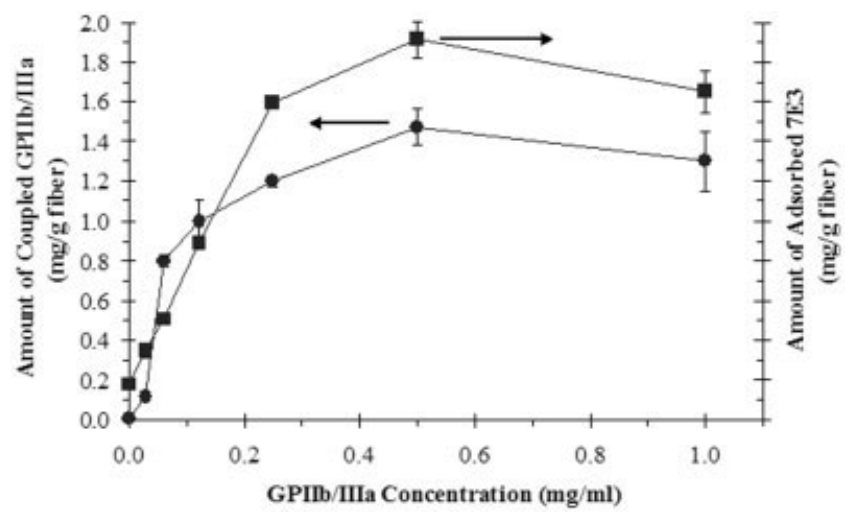

Figure 6. Effect of GPIIb/IIIa concentration on coupling of GPIIb/IIIa. Experımental details were provided in the Methods section. All measurements were performed in triplicate. Error bars represented standard deviation. (O) represents the amount of coupled GPIIb/IIIa, whereas ( $\square$ ) represents the amount of adsorbed 7E3. 
Figure 6 show that the adsorption capacity of the bioreactor toward 7E3 increased with the increase of the GPIIb/IIIa loading in these bioreactors. A maximum 7E3 adsorption capacity of $1.90 \mathrm{mg} / \mathrm{g}$ fiber was observed for the bioreactor containing the maximum loading of $1.46 \mathrm{mg}$ GPIIb/IIIa/g fibers. In comparison, the nonspecific 7E3 adsorption onto a sham bioreactor was only $0.17 \mathrm{mg} / \mathrm{g}$ fibers.

Desorption of 7E3 from the GPIIb/IIIa bioreactor was also examined. Up to $93 \%$ of the adsorbed 7E3 was recovered from the bioreactor after four cycles of washings using $0.1 \mathrm{M}$ aminoacetic acid as the eluting agent. The elution of the bioreactor by aminoacetic acid likely changed the charges or, in other words, the isoelectric point of GPIIb/IIIa side groups, resulting in the detachment of 7E3 from the bioreactor. It should be noted that there was no detectable GPIIb/IIIa release from the bioreactor, indicating that GPIIb/IIIa molecules were stably and covalently immobilized to the epichlorohydrin-activated hollow fibers. With these desorption results, it was concluded that aminoacetic acid was a suitable agent to regenerate an already used GPIIb/IIIa bioreactor.

Stability and reusability of the GPIIb/IIIa bioreactor

To evaluate the stability and shelf-life of the bioreactor, leakage of GPIIb/IIIa from the bioreactor was examined by filling the interior of the bioreactor with PBS buffer ( $\mathrm{pH} 7.4$ ) and then storing it at room temperature for 4 days. At specific time intervals, $100 \mu \mathrm{L}$ of the filling solution was withdrawn to determine the degree of leakage of GPIIb/IIIa. Data showed that, on average, $<1 \%$ of the initially loaded GPIIb/IIIa was leached off from the bioreactor after each day of storage, and a total of 3\% of the initially loaded GPIIb/IIIa were lost during the entire span of 4 days of storage. Hence, the GPIIb/IIIa bioreactor thus prepared appears to possess a reasonable shelf-life for its potential clinical application.

To evaluate the reusability of the GPIIb/IIIa bioreactor, the 7E3 adsorption/desorption cycle discussed above was repeated six times over a period of 20 days (i.e., one cycle every 4 days) with the same bioreactor. Results showed that the capacity of the bioreactor toward 7E3 adsorption was reduced by an approximate rate of $2 \%$ per cycle of reuse. At the 20 -day mark, $87 \%$ of the initial 7E3 adsorption capacity of the bioreactor remained.

To further assess the reusability of the GPIIb/IIIa bioreactor on a daily basis, the adsorption-desorption cycle was repeated three times over a 1-day period with the same bioreactor. Results showed that there was no statistically significant reduction in the adsorption capacity of the GPIIb/IIIa bioreactor. By compounding these findings together, it was concluded that the reduction in adsorption capacity of the biore-

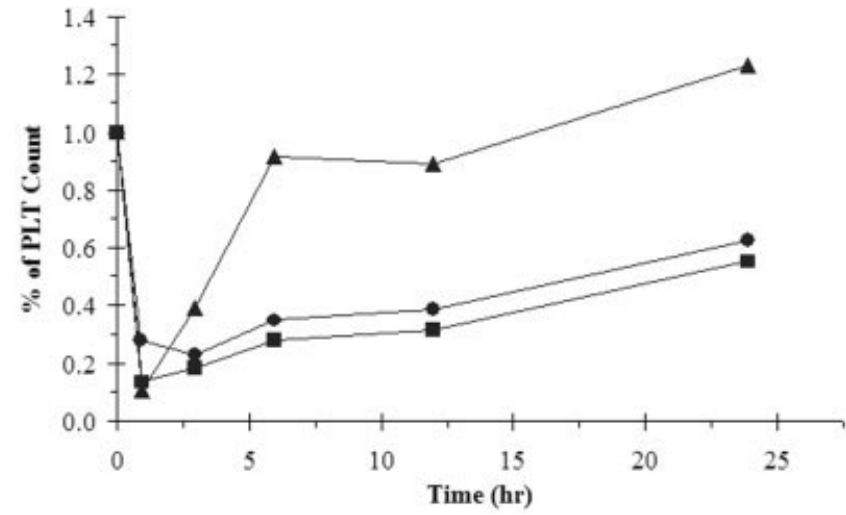

Figure 7. Platelet count in 7E3-injected rats treated with the albumin and GPIIb/IIIa bioreactors. (O) represents the control group in which the rat was attached with a sham bioreactor, $(\boldsymbol{\Delta})$ represents the experimental in which the rat was attached with a GPIIb/IIIa bioreacotr, and ( $\square$ ) represents another control group in which the rat was attached with an albumin-linked bioreactor.

actor was probably less likely due to the experimental conditions during its usage, but more to do with the storage time.

\section{Preliminary in vivo proof-of-concept studies in rats}

Very preliminary proof-of-concept type of investigation was carried out on rats to show the feasibility of the bioreactor approach in treating ITP. Thrombocytopenia was induced in rats by administration, via the jugular vein cannula of the animal, of a dose of $4 \mathrm{mg} / \mathrm{kg}$ of 7E3. The ITP-induced rats were then distributed into three subgroups (three rats per group): 1) control group in which the rats were connected with a sham bioreactor; 2 ) experimental group in which the rats were connected with a GPIlb/IIIa bioreactor; and 3) another control group in which the rats were connected with an albumin-linked bioreactor to determine the degree of nonspecific adsorption of 7E3 onto the hollow fiber-based device. Extracorporeal circulation through the bioreactor was initiated $1 \mathrm{~h}$ after administration of 7E3. Figure 7 presents a representative picture that illustrates the trend of changes in platelet counts in animals in response to the bioreactor treatment. As seen, $1 \mathrm{~h}$ after 7E3 injection, PLT counts in all of the animals were reduced to $15-25 \%$ of their initial values, a typical 7E3-induced thrombocytopenic response in rats as reported elsewhere. ${ }^{36}$ In both control groups, the PLT counts continued to remain exceedingly low over at least $12 \mathrm{~h}$ and then started to slightly rebound gradually. These results suggested a sustained PLT destruction due to the presence of the 7E3 antibodies. It appeared that there was very limited nonspecific adsorption of 7E3 on the hollow fiber-based bioreactor, because the albumin-coated bioreactor yielded similar PLT destruction as that of the sham bio- 
reactor. In sharp contrast, rats that received the GPIIb/ IIIla bioreactor treatment displayed a remarkably rapid rebound in PLT counts, because the PLT count completely returned to the initial healthy level that was measured before any bioreactor treatment (Fig. 7).

Measurements of the blood-free 7E3 concentrations of the treated animals by using the ELISA assay yielded comparable results. Five hours after the bioreactor treatment, 7E3 concentrations in the sham and albumin bioreactor-treated groups were reduced by $20 \%$ and $25 \%$, respectively. In contrast, the 7E3 concentration in the GPIIb/IIIa bioreactor-treated rats was markedly reduced by $50 \%$. At the 24 -h mark after the treatment, approximately $80 \%$ of the administered 7E3 was removed by the GPIIb/IIIa bioreactor. By correlating these results with those seen in Figure 7 in the change of PLT counts, it is obvious that specific removal of the 7E3 antibodies would significantly retard the destruction of PLT, thereby providing a useful means to combat ITP.

It should be pointed out that, in practice, the use of an extracorporeal GPIIb/IIIa bioreactor could never completely (i.e., 100\%) deplete the 7E3 autoantibodies produced in ITP. This is because the extracorporeal circuit only represents one of the many branches involved in blood circulation in the body. Hence, when the bioreactor-filtered blood returns to the patient, this specific 7E3free bloodstream will be mixed with blood from other branches that contain 7E3. As a consequence, the concentration of 7E3 can only be reduced to a minimum value regardless of the GPIIb/IIIa bioreactor efficiency and number of blood circulation cycles. On the other hand, based on the preliminary results shown in Figure 7 , it may not be necessary to achieve a 100\% antiplatelet antibody removal.

To examine the hematological effects of the bioreactor treatment, the red blood cell (RBC) count, white blood cell (WBC) count, and total hemoglobin content in the treated animals were analyzed. As expected, the RBC count and total hemoglobin content remained virtually unchanged over the entire course of experiments for all of the three animal groups. It is of interest that the WBC counts in both the control groups (i.e., animals treated with a sham or an albumin bioreactor) decreased to about $20 \%$ of their initial values $1 \mathrm{~h}$ after the treatment and then returned to the initial values approximately $9 \mathrm{~h}$ after the treatment. On the other hand, WBC counts in the GPIIb/IIIa-treated rats were only reduced to $50 \%$ of their initial values $1 \mathrm{~h}$ after the treatment and then rebounded rapidly to the initial values within a mere $5 \mathrm{~h}$ after the GPIlb/IIIla treatment.

\section{CONCLUSIONS}

Given the evidence that antiplatelet antibodies $(\mathrm{APAb})$ are responsible for the increased rate of PLT destruction observed in ITP, it is of no surprise that recently increased attention has been given to approaches that will lead to an accelerated removal of these pathogenic antibodies. Indeed, the immunoadsorptive plasmapheresis method by using the PROSORBA column has been approved by the FDA for clinical trials. ${ }^{24,25}$ Despite some clinical promise, a recent ITP "practice guideline" prepared by a panel of the American Society of Hematology listed Protein-A therapy for refractory ITP patients (i.e., refractory to prednisone and splenectomy) as a low preference therapy compared with IVIG. ${ }^{1}$ This low preference given to Protein-A plasmapheresis was apparently attributed to the nonspecific nature of this therapy; all IgG would be extracted from plasma, thereby increasing the patient's susceptibility to opportunistic infections. To this regard, the proposed approach possesses the potential to overcome all these shortcomings associated with the PROSORBA approach concomitantly. First, the GPIIb/IIIa bioreactor will specifically remove $\mathrm{APAb}$, and thus patients will not be immunocompromised through removal of nonpathogenic IgG. Second, the new system does not use Protein-A or silica, two components that have been known to cause thrombosis after their leaching from the PROSORBA column and into the patient's blood stream. Third, unlike the PROSORBA device that is established on silica gels, the proposed system is built on cellulose hollow fibers. A hollow fiber-based bioreactor offers unmatched advantages over a particulate-based device because it is nonresistant to blood flow and does not require separation of blood components during its operation.

Overall, the preliminary findings presented in this article were extremely promising, implicating that the development of a highly improved new alternative in treating ITP is reasonably plausible. Continued studies to further optimize the loading and adsorption capacity of this GPIIb/IIIa bioreactor, as well as to confirm the real-time feasibility of this approach via extensive animal testing, are currently underway in our laboratory.

The authors thank Feng Jin, Department of Pharmaceutics, State University of New York at Buffalo, for his technical assistance during the in vivo rat studies.

\section{References}

1. George JN, Woolf SH, Raskob GE, Wasser JS, Aledort LM, Ballem PJ, Blanchette VS, Bussel JB, Cines DB, Kelton JG, et al. Idiopathic thrombocytopenic purpura: a practice guideline developed by explicit methods for the American Society of Hematology. Blood 1996;88:3-40.

2. Harington WJ, Ninnich V, Hollingsorth JW, Moore CB. Demonstration of thrombocytopenic factor in the blood of patients with thrombocytopenic purpura. J Lab Clin Med 1951;38. 
3. McMillan R. Therapy for adults with refractory chronic immune thrombocytopenic purpura. Ann Intern Med 1997;126: 307-314.

4. Imbach P, Dapuzzo V, Hirt A, Rossi E, Vest M, Barandun S, Baumgartner C, Morell A, Schoni M, Wagner HP. High-dose intravenous gamma-globulin for idiopathic thrombocytopenic purpura in childhood. Lancet 1981;1:1228-1231.

5. Salama A, Muellereckhardt C, Kiefel V. Effect of intravenous immunoglobulin in immune thrombocytopenia-competitive inhibition of reticuloendothelial system function by sequestration of autologous red blood cells. Lancet 1983;2:193-195.

6. Newland AC, Treleaven JG, Minchinton RM, Waters AH. High-dose intravenous Igg in adults with autoimmune thrombocytopenia. Lancet 1983;1:84-87.

7. Bussel JB, Kimberly RP, Inman RD, Schulman I, Cunninghamrundles C, Cheung N, Smithwick EM, Omalley J, Barandun S, Hilgartner MW. Intravenous gamma-globulin treatment of chronic idiopathic thrombocytopenic purpura. Blood 1983;62: 480-486.

8. Imbach P, Berchtold W, Hirt A, Muellereckhardt C, Rossi E, Wagner HP, Gaedicke G, Joller P, Muller B, Barandun S. Intravenous immunoglobulin versus oral corticosteroids in acute immune thrombocytopenic purpura in childhood. Lancet 1985; 2:464-468.

9. Blanchette VS, Kirby MA, Turner C. Role of intravenous immunoglobulin- $\mathrm{G}$ in autoimmune hematologic disorders. Semin Hematol 1992;29:72-82.

10. Hollenberg JP, Subak LL, Ferry JJ, Bussel JB. Cost-effectiveness of splenectomy versus intravenous gamma-globulin in treatment of chronic immune thrombocytopenic purpura in childhood. J Pediatr 1988;112:530-539.

11. Bussel JB, Pham LC, Aledort L, Nachman R. Maintenance treatment of adults with chronic refractory immune thrombocytopenic purpura using repeated intravenous infusions of gamma-globulin. Blood 1988;72:121-127.

12. Nydegger UE, Sturzenegger M. Adverse effects of intravenous immunoglobulin therapy. Drug Saf 1999;21:171-185.

13. Ahsan N. Intravenous immunoglobulin-induced nephropathy: a complication of IVIG therapy. J Nephrol 1998;11:157-161.

14. Cayco AV, Perazella MA, Hayslett JP. Renal insufficiency after intravenous immune globulin therapy: a report of two cases and an analysis of the literature. J Am Soc Nephrol 1997;8: 1788-1794.

15. Mathy I, Gille M, Van Raemdonck F, Delbecq J, Depre A. Neurological complications of intravenous immunoglobulin (IVIg) therapy: an illustrative case of acute encephalopathy following IVIg therapy and a review of the literature. Acta Neurol Belg 1998;98:347-351.

16. Healey CJ, Sabharwal NK, Daub J, Davidson F, Yap PL, Fleming KA, Chapman RWG, Simmonds P, Chapel H. Outbreak of acute hepatitis $C$ following the use of anti-hepatitis $C$ virusscreened intravenous immunoglobulin therapy. Gastroenterology 1996;110:1120-1126.

17. Widell A, Zhang YY, AnderssonGare B, Hammarstom L. At least three hepatitis $C$ virus strains implicated in Swedish and Danish patients with intravenous immunoglobulin-associated hepatitis C. Transfusion 1997;37:313-320.

18. Taliani G, Guerra E, Rosso R, Badolato MC, Luzi G, Sacco G, Lecce R, Debac C, Aiuti F. Hepatitis-C virus infection in hypogammaglobulinemic patients receiving long-term replacement therapy with intravenous immunoglobulin. Transfusion 1995; 35:103-107.
19. Guthrie TH, Oral A. Immune thrombocytopenia purpura-a pilot study of staphylococcal protein-A immunomodulation in refractory patients. Semin Hematol 1989;26:3-9.

20. Rice L, Nichol JL, McMillan R, Roskos LK, Bacile M. Cyclic immune thrombocytopenia responsive to thrombopoietic growth factor therapy. Am J Hematol 2001;68:210-214.

21. Cines DB, McKenzie SE, Siegel DL. Mechanisms of action of therapeutics in idiopathic thrombocytopenic purpura. J Pediatr Hematol Oncol 2003;25:S52-S56.

22. Scaradavou A, Woo B, Woloski BMR, Cunningham-Rundles $\mathrm{S}$, Ettinger LJ, Aledort LM, Bussel JB. Intravenous anti-D treatment of immune thrombocytopenic purpura: experience in 272 patients. Blood 1997;89:2689-2700.

23. Salama A, Kiefel V, Muellereckhardt C. Effect of Igg antirho(D) in adult patients with chronic autoimmune thrombocytopenia. Am J Hematol 1986;22:241-250.

24. Muroi K, Sasaki R, Miura Y. The effect of immunoadsorption therapy by a protein-A column on patients with thrombocytopenia. Semin Hematol 1989;26:10-14.

25. Snyder HW, Bertram JH, Channel M, Ernst NR, Balint JP, Jones FR. Reduction in platelet-binding immunoglobulins and improvement in platelet counts in patients with HIV-associated idiopathic thrombocytopenia purpura (Itp) following extracorporeal immunoadsorption of plasma over staphylococcal protein-A-silic. Artif Organs 1989;13:71-77.

26. Kondo A, Teshima T. Preparation of immobilized enzyme with high-activity using affinity tag based on protein-A and protein-G. Biotechnol Bioengineer 1995;46:421-428.

27. Kabisch A, Kroll H, Wedi B, Kiefel V, Pralle H, Muellereckhardt $\mathrm{C}$. Severe adverse effects of protein-A immunoadsorption. Lancet 1994;343:116-116.

28. Dzik WH, Duncan LM, Robertson R. A 55-year-old woman with a skin rash and hemiparesis after staphylococcal protein-A column therapy-leukocytoclastic vasculitis, due to staphylococcal protein-A column therapy-(cerebral infarction of undetermined pathogenesis). N Engl J Med 1994;331:792800.

29. Vanleeuwen EF, Vanderven JTM, Engelfriet CP, Vondemborne AEGK. Specificity of autoantibodies in autoimmune thrombocytopenia. Blood 1982;59:23-26.

30. Kucera J. Preparation of cellulose derivatives for affinity chromatography and immobilization of enzymes-activation by epichlorohydrin. Collect Czech Chem Commun 1979;44:804807.

31. Matsumoto I, Mizuno Y, Seno N. Activation of sepharose with epichlorohydrin and subsequent immobilization of ligand for affinity adsorbent. J Biochem 1979;85:1091-1098.

32. Nagasawa J, Suehiro T, Yamauchi A. Immobilization of DNA via covalent linkage for use as immunosorbent. J Appl Biochem 1985;7:296-302.

33. Bradford MM. Rapid and sensitive method for quantitation of microgram quantities of protein utilizing principle of proteindye binding. Anal Biochem 1976;72:248-254.

34. Hansen RJ, Balthasar JP. An ELISA for quantification of murine IgG in rat plasma: application to the pharmacokinetic characterization of AP-3, a murine anti-glycoprotein IIIa monoclonal antibody, in the rat. J Pharm Biomed Anal 1999;21:1011-1016.

35. Scoble J, Scopes R. Well defined dye adsorbents for protein purification. J Mol Recog 1996;9:728-732.

36. Hansen RJ, Balthasar JP. Pharmacokinetics, pharmacodynamics, and platelet binding of an anti-glycoprotein IIb/IIIa monoclonal antibody (7E3) in the rat: a quantitative rat model of immune thrombocytopenic purpura. J Pharmacol Exp Ther 2001;298:165-171. 